\title{
De Cabanons en cabanes
}

Habitat pastoral et retour du loup. L'élevage de Crau pour exemple

From hut to cabin pastoral housing and the return of the wolf. The example of sheep breeding in the Crau

\section{Marc Vincent et Lucie Dupré}

\section{(2) OpenEdition Journals}

Édition électronique

URL : https://journals.openedition.org/tc/5675

DOI : $10.4000 /$ tc. 5675

ISBN : $1952-420 X$

ISSN : 1952-420X

Éditeur

Éditions de l'EHESS

\section{Édition imprimée}

Date de publication : 5 septembre 2011

Pagination : 114-131

ISBN : 978-2-7351-1410-8

ISSN : 0248-6016

Référence électronique

Marc Vincent et Lucie Dupré, « De Cabanons en cabanes », Techniques \& Culture [En ligne], 56 | 2011, mis en ligne le 30 novembre 2011, consulté le 29 septembre 2022. URL : http://

journals.openedition.org/tc/5675; DOI : https://doi.org/10.4000/tc.5675 


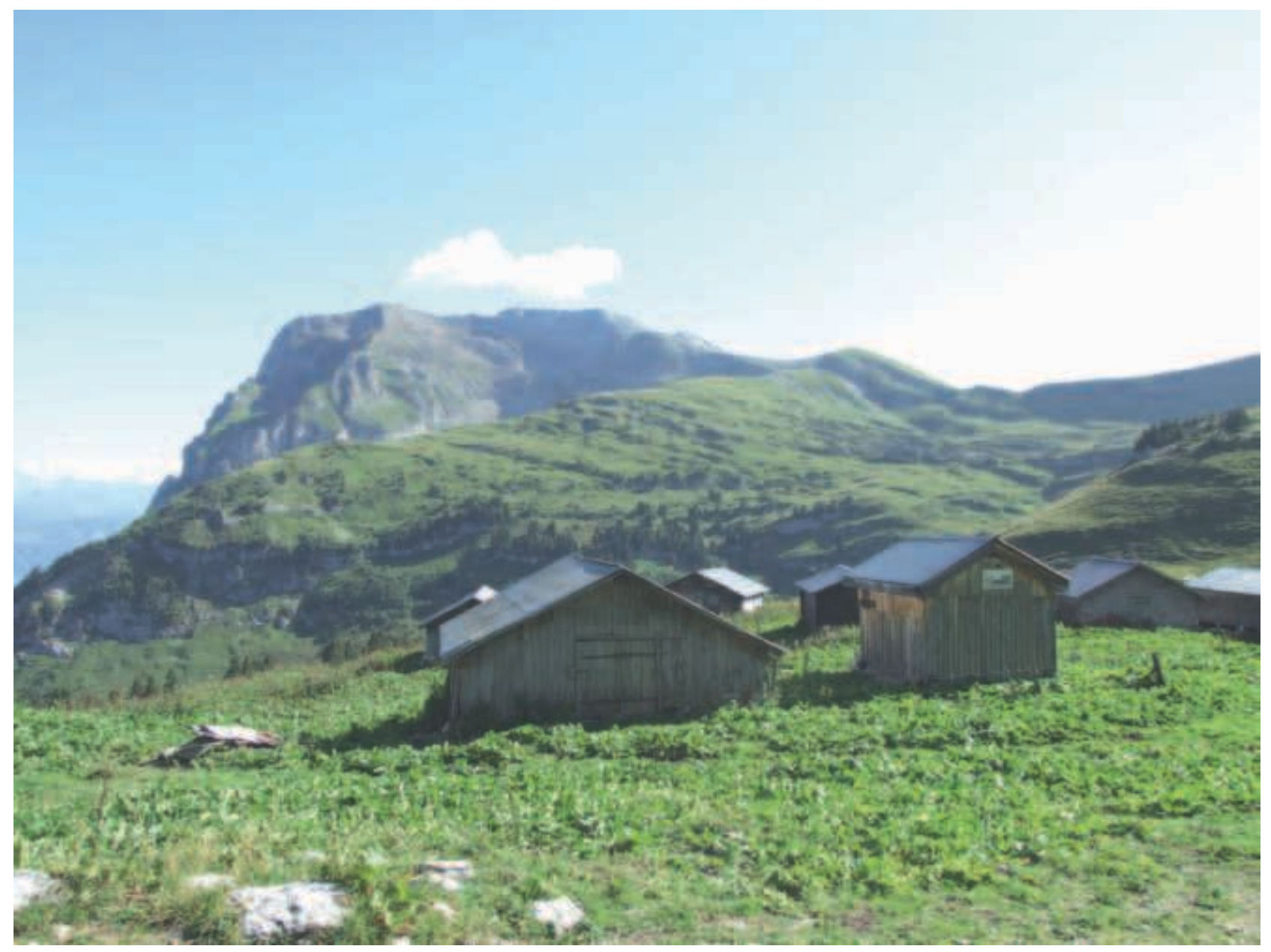

๑ M. Vincent 


\section{DE CABANONS EN CABANES}

\section{Habitat pastoral et retour du loup : l'élevage de Crau pour exemple}

Le conflit biblique qui déchire Caïn et Abel oppose deux grands types d'activité agricole déployant chacune un point de vue sur le monde et ses ressources: l'agriculture proprement dite (c'est-à-dire le cultivateur en la personne de Caïn) et le pasteur (le berger Abel). Ces deux archétypes renvoient à deux rapports conflictuels au territoire: la sédentarité d'un côté, la mobilité de l'autre, et s'accompagnent d'un type d'habitat nécessairement différent dont cette contribution examine l'une des formes contemporaines liées à la transhumance. La réflexion trouve sa place dans la mosaïque disciplinaire de ce dossier en étant lui-même le produit d'une collaboration ad hoc entre un pastoraliste qui questionne l'évolution du pastoralisme en contexte de prédation, et d'une ethnologue engagée dans une réflexion autour de l'évolution des conditions d'exercice des métiers agricoles. Elle contribue à documenter l'une des grandes lignes de force structurant ce volume: l'articulation entre pratiques d'habitat et exercice d'une activité professionnelle d'aujourd'hui qui s'appuie sur l'exploitation de ressources naturelles stratégiques liées à des territoires hors norme, isolés et difficiles.

Le déplacement répété des troupeaux et des hommes tient à un système fourrager reposant sur des ressources consommées sur pied. Lorsque ces dernières sont épuisées et que les cycles climatiques ne permettent pas de séjourner davantage au même endroit, les bergers conduisent leurs troupeaux vers des pâturages d'altitude, ou vers des zones plus humides, où de nouvelles ressources sont disponibles. Ces migrations temporaires prennent des formes et des amplitudes différentes selon les cultures pastorales: elles peuvent concerner un seul berger ou l'ensemble du groupe, et plusieurs espèces (chameaux, rennes, chèvres, vaches, brebis). Même remises en cause ou réaménagées, réduites et fragilisées, elles structurent fortement calendrier et territoires pastoraux et constituent la clé de voûte de 


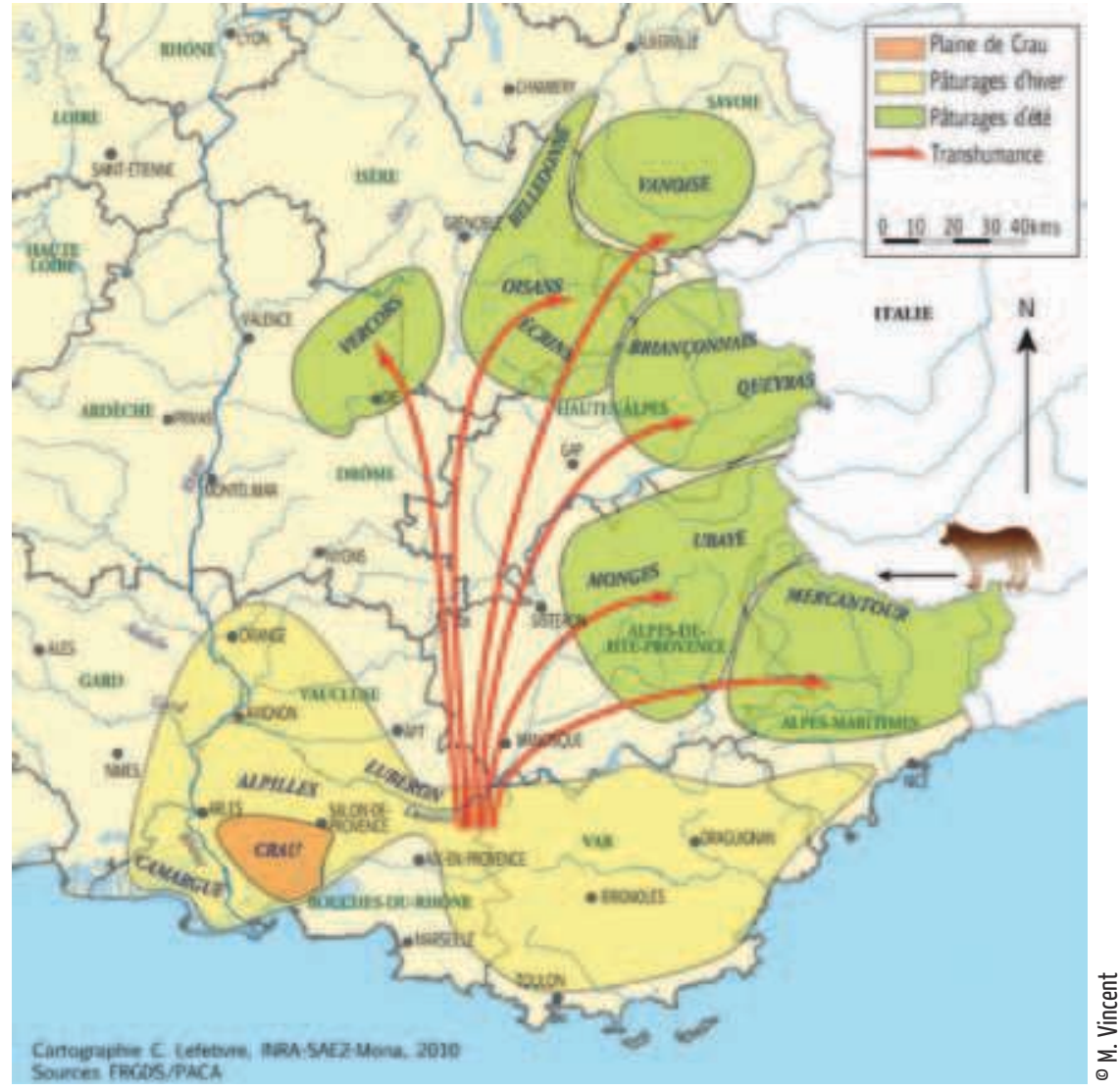

Les trajets de la grande transhumance de la plaine vers les alpages, où des loups en provenance d'Italie se sont installés depuis 1992.

(Fig. 1) nombreux systèmes d'élevage, au Nord comme au Sud (Faye 2008). L'itinérance pastorale impose un type d'habitat adapté: dans le cas du nomadisme, le logement qui suit famille et troupeau est reconstitué à chaque étape; en ce qui concerne la transhumance, l'habitat est permanent mais utilisé de façon temporaire par les bergers.

La question de l'habitat apparaît centrale dans le nomadisme en tant qu'elle concerne l'ensemble du groupe social dont elle exprime et structure les valeurs (Claudot-Hawad 2002). Elle n'est toutefois que rarement abordée dans l'analyse des systèmes pastoraux itinérants européens, généralement ovins. Pourtant, dans le Sud-Est de la France par exemple, le pastoralisme est fondé sur ce même principe fourrager; fortement structuré autour de la transhumance, conduisant bêtes et hommes des pâturages d'hiver situés en plaine vers les estives de montagne, il impose une réelle mobilité résidentielle, aussi bien en montagne qu'en plaine. L'élevage de moutons caractéristique du delta rhodanien (Crau/Camargue) est à ce titre exemplaire de ce système d'élevage. Mais si de nombreux travaux et documents concernent la transhumance, période devenue emblématique, les problèmes de l'habitat pastoral y sont rarement évoqués. On peut avancer deux raisons. D'une part, les analyses consacrées au pastoralisme provençal privilégient généralement les savoirs et les savoir-faire ainsi que le rapport aux ressources naturelles (Brisebarre 2007); la cabane ne devenant visible que lorsqu'elle émarge à la catégorie de patrimoine architectural, ce qui n'est pas toujours le cas dans l'arc alpin. D'autre part, les réflexions dédiées au statut et à l'identité professionnelle du berger n'accordent généralement pas une place centrale à l'habitat alors qu'il s'agit d'une pièce maîtresse dans le travail pastoral en montagne, plus encore depuis que les bergers sont confrontés à un problème de taille: les attaques de loups.

La figure du vieux berger provençal drapé dans sa solitude et campé aux confins de la société, a fait son temps. Le métier a changé plus rapidement et plus profondément sans doute que les images encore tenaces d'archaïsme qui lui sont associées. Un diplômé salarié est en train de se substituer à l'ancien cadet de famille, « berger malgré lui ». Son rôle et son métier, ses droits et ses conditions de vie et de travail sont appelés, eux aussi, à suivre le même mouvement. Ces transformations sont difficiles à dater car elles relèvent d'un faisceau d'événements aux conséquences plus ou moins directes. En revanche, 
l'année 1992 a marqué l'histoire du pastoralisme méditerranéen, dont un événement a brutalement modifié le cours. Une espèce animale strictement protégée est venue d'Italie, a fait son retour dans les Alpes françaises d'où elle avait disparu au début du xx ${ }^{e}$ siècle: Canis lupus, le loup. Les bergers se sont trouvés complètement désarmés face au prédateur dont les premières attaques - puis leur éventualité - ont brutalement bouleversé la montagne. La colère et le découragement ont succédé à l'impuissance et au désarroi des bergers aujourd'hui tenus de cohabiter avec les deux cents loups répartis dans l'ensemble du massif et en particulier dans les zones d'alpage vers lesquelles les moutons de la Crau - et du Sud-Est de la France en général (Figure 1) - transhument aux beaux jours (Vincent 2011). C'est dans ce contexte très particulier que nous nous intéressons à l'habitat pastoral dans le système ovin transhumant de la Crau que nous présenterons à grands traits. Nous reviendrons ensuite sur les différents logements occupés successivement par les bergers tout au long de l'année, en nous focalisant particulièrement sur l'habitat en montagne. Enfin, nous montrerons en quoi la présence du loup rend encore plus décisive et stratégique la double fonction, résidentielle et pastorale, des cabanes d'alpage dont une variante exigée par la situation commence à s'installer dans le paysage.

\section{De la plaine aux alpages: hommes et bêtes toujours en mouvement}

Dans le Sud-Est de la France, l'élevage de moutons est très ancien comme en attestent en plaine et en montagne des vestiges d'infrastructures pastorales. Les plus anciennes datent du néolithique et sont des grottes bergeries situées en alpage (voir dans ce numéro la contribution de Alain Beeching). Des restes de bergeries romaines suggèrent une exploitation systématique de la Crau par l'élevage ovin depuis le début de notre ère jusqu'au v viècle (Badan \& al. 1996). Dans cette plaine, l'élevage ovin est particulièrement

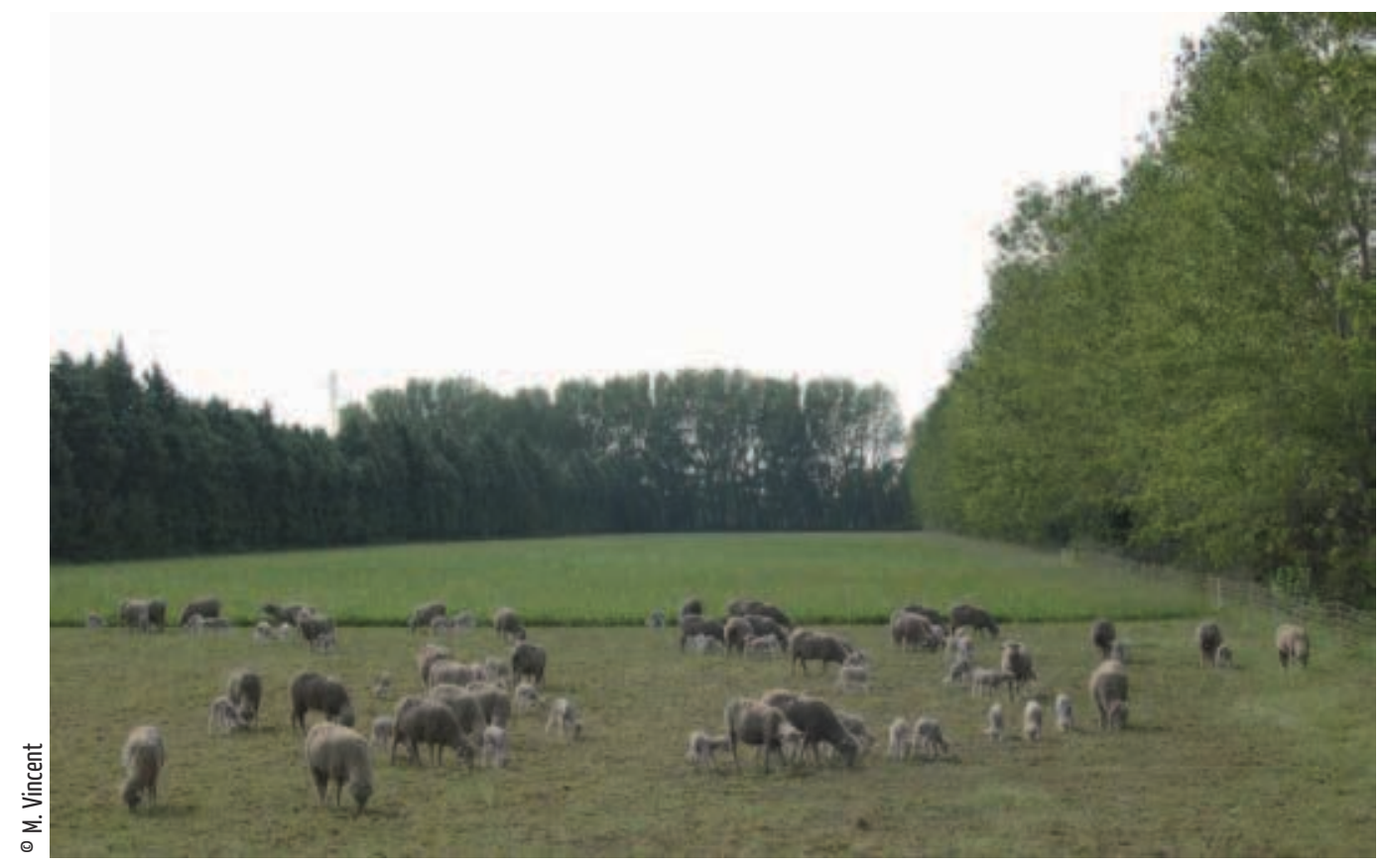

Brebis et agneaux dans une parcelle de regain de prairie de Crau ou « 4e coupe » (Bouches-du-Rhône)

Grâce à l'irrigation des prairies naturelles, la ressource fourragère, de qualité et toujours disponible, est adaptée à la fin de gestation et à la lactation. (Fig. 2) 


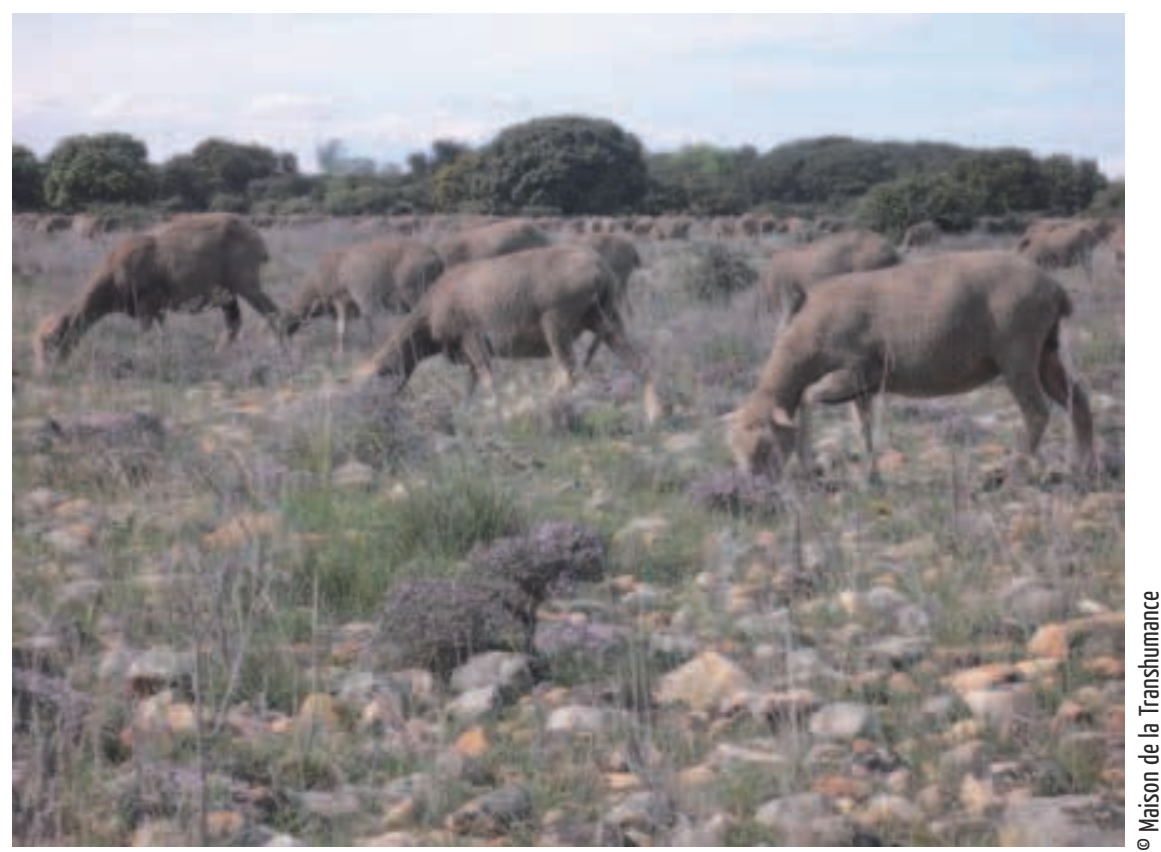

\section{Troupeau au pâturage d'hiver dans le coussou/de Crau}

La végétation peu dense convient à des brebis taries; le mistral pendant l'hiver en fait un milieu difficile pour le berger.

(Fig. 3) ancien, mais ce système original a su évoluer au fil du temps, notamment de la production de la laine vers celle de la viande (Fabre \& Lebaudy 2010). Aujourd'hui le Pays d'Arles (Crau et Camargue) compte les plus gros troupeaux de France $^{1}$. Les activités d'élevage sont organisées de différentes façons. Certains éleveurs sont propriétaires de pâturages, mais d'autres sont des éleveurs sans terre appelés localement « herbassiers ». Propriétaires du seul cheptel, les herbassiers doivent acheter chaque année des ressources fourragères sur pied - c'est ce qu'on appelle une « place »-sans aucune garantie de pouvoir en disposer l'année suivante en l'absence de bail établi. En plaine, l'éleveur garde parfois ses propres bêtes - c'est le cas de la plupart des « herbassiers »; il les confie souvent à un berger salarié, éventuellement employé à l'année, mais qui peut être un saisonnier, notamment pour la période d'estive. Dans le Sud-Est de la France, le pastoralisme occupe - c'est-à-dire exploite et valorise - de vastes territoires complémentaires: la plaine en hiver et la montagne en été (Figure 1), chacun étant associé à un type de pâturage bien spécifique.

En plaine de Crau, l'automne est la période de l'agnelage ${ }^{2}$ : le troupeau trouve dans le regain des prairies l'alimentation riche et abondante exigée par le stade physiologique (fin de gestation et lactation) (Figure 2). Cette herbe est le co-produit du foin de Crau, première production agricole à vocation fourragère ayant obtenu une Appellation d'Origine Contrôlée (1997). Le cahier des charges imposant trois coupes au maximum, le regain ou « $4^{e}$ coupe » est vendu sur pied aux éleveurs. À cette période intense d'élevage succède l'hivernage. Les bêtes se déplacent vers le coussoul voisin, milieu steppique où la nourriture est maigre mais adaptée à des brebis à l'entretien ${ }^{3}$ (Figure 3). Les éleveurs qui n'y ont pas accès gagnent les collines provençales et leurs broussailles (Figure 4). À la fin du printemps, c'est la transhumance. Le troupeau rejoint en camion les massifs alpins où il va demeurer jusqu'à l'automne sous la garde d'un berger. Bien que le travail y soit éprouvant et intense, le séjour en montagne constitue une étape cruciale; éleveurs et bergers le considèrent comme des « vacances » pour eux et le troupeau. Après le dur hivernage, l'alpage offre en effet un ressourcement pour tous. Le stade physiologique du troupeau y est pour beaucoup : la plupart des brebis sont en gestation et l'art du berger consiste à utiliser au mieux l'espace montagnard et ses ressources fourragères pour leur permettre de reprendre du poids et de préparer leur lactation. Le territoire d'alpage affecté à un troupeau et son berger s'appelle estive, montagne, ou encore, dans un langage plus administratif, unité pastorale. Il se divise en quartiers, secteurs de pâturage situés à différentes altitudes où la végétation arrive 
à maturité de façon échelonnée. Une montagne compte ainsi des quartiers bas, les quartiers dits de « juillet », pâturés en début et en fin de saison et des quartiers hauts, ou « d'août ».

\section{L'environnement: une nouvelle assise professionnelle}

À partir des années 1990, s’amorce un tournant majeur dans l'histoire du pastoralisme: dans sa refonte radicale, la politique agricole européenne prend désormais en compte la protection de l'environnement, jusqu'alors nettement tenue à l'écart des questions agricoles. L'élevage ovin est particulièrement concerné dans la mesure où il trouve ainsi l'occasion d'officialiser son rôle dans la préservation d'espèces et d'espaces patrimoniaux, tant dans les territoires pastoraux de plaine que de montagne. La Crau et son coussoul en sont un cas d'école. Delta fossile de la Durance, la Crau conserve une zone originelle steppique, le coussoul. Cette steppe riche en galets de rivière abrite une biodiversité unique en France en termes d'espèces et d'habitats d'espèces (outarde, ganga, oedicnème, ...) dont la richesse, liée à un pâturage répété et très ancien, ne pourrait se maintenir sans la présence régulière des moutons (Conservatoire études et écosystèmes de Provence \& Chambre d'agriculture des Bouches-du-Rhône 1997). Le pastoralisme « rend » par ailleurs des « services » à la collectivité. Par exemple, le pâturage en colline empêche la banalisation et la fermeture de ce milieu embroussaillé très inflammable et facilite la lutte contre les incendies. En montagne, les troupeaux contribuent à nettoyer de vastes surfaces enherbées, domaine skiable l'hiver, dont ils assurent l'entretien en prévenant le risque d'avalanches. Dans ces espaces de plaine, de colline, comme de montagne, l'État passe des contrats rémunérés, dit contrats agri-environnementaux, avec des éleveurs qui s'engagent à mettre en œuvre des pratiques de pâturage (obligation de moyens) répondant à des objectifs de conservation précis: la mise en défends de zones humides, le pâturage tardif pour aider la nidification d'espèces sensibles (outardes, tétraslyre), un niveau de raclage optimum de l'herbe pour limiter le risque d'incendie, la gestion fine en parcs mobiles ou en gardiennage serré de pelouses dégradées (nard, queyrel). Le berger doit alors appliquer un plan de pâturage élaboré, en concertation, par l'éleveur et le gestionnaire du site et intégrant ces différentes clauses.

Ces politiques publiques ont offert, particulièrement en alpage, une nouvelle assise au métier de berger transhumant. Dans le même temps, elles ont contribué à redéfinir ce métier sur la base d'une légitimité environnementale. En montagne, à la 


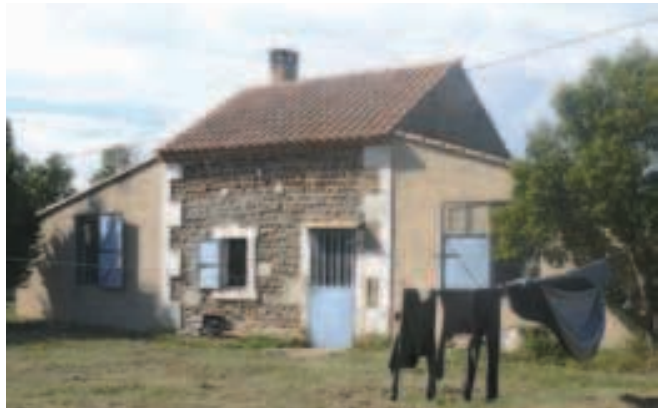

${ }^{\circ}$ M. Vincent - Fig. 5

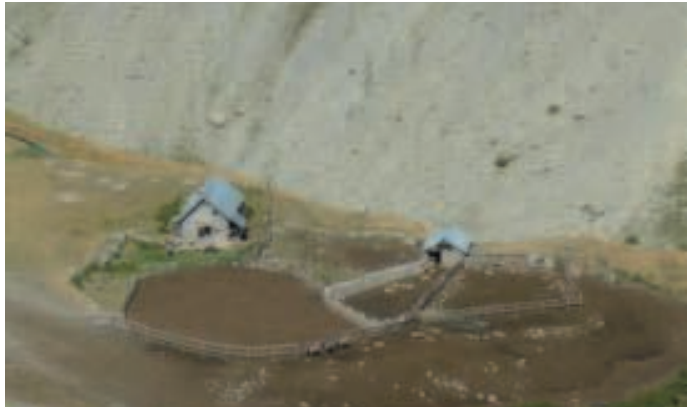

${ }^{\circ}$ M. Vincent - Fig. 6

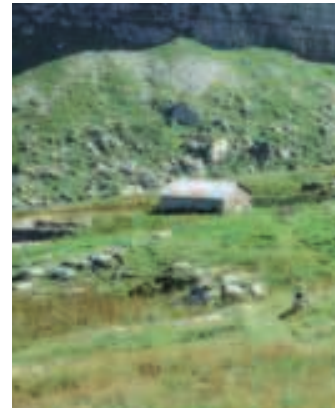

${ }^{\circ}$ M. Vincent - Fig. 7

\section{Cabanon de Crau situé au cœur des prairies}

Ce logement est attenant à la bergerie pour faciliter la surveillance de l'agnelage. (Fig. 5)

\section{Cabane principale dans les Alpes-de-Haute-Provence}

À noter, l'important système de contention du troupeau et la présence d'ânes de bât indispensables au ravitaillement. (Fig. 6)

\section{Cabane principale en Haute-Savoie}

Certains alpages de ce département alpin sont aujourd'hui repris par des transhumants après le départ des éleveurs laitiers locaux. Le pâturage empêche la fermeture rapide du milieu colonisé notamment par des ligneux puissants comme l'aulne vert (Alnus viridis). (Fig. 7) fois guide du troupeau, gestionnaire des ressources alimentaires, vétérinaire, le berger est aussi chargé de l'entretien des équipements pastoraux (cabanes, parcs, pédiluve...), de l'application des contrats agri-environnementaux et de la protection du troupeau contre les prédateurs, mais aussi des interfaces avec les autres usagers de la montagne, de plus en plus nombreux. Des écoles spécialisées - comme celle du Domaine du Merle à Salon-de-Provence - forment chaque année des salariés qualifiés dans le travail d'alpage. Les bergers d'aujourd'hui sont plus jeunes et la profession se féminise; les bergers ont fait le choix d'un métier difficile et revendiquent la possibilité de l'exercer dans de bonnes conditions de travail et de vie (Jallet \& Fabre 2007; Legeard 1996; Legeard \& al. 2010). Responsabilités accrues et qualifications poussées sont autant d'indices de l'évolution de la profession.

\section{De cabanon en cabanes: la mobilité résidentielle}

De la plaine à l'alpage, les bergers s'installent successivement dans plusieurs logements - jusqu'à trois ou quatre sur l'année ${ }^{4}$. L'habitat pastoral est mis à la disposition du berger par l'éleveur; il est généralement en dur (plus rarement mobile) et relève schématiquement de deux grandes catégories: le cabanon en plaine et la cabane en alpage.

Dans le vocabulaire agricole, le cabanon est une « petite construction dans les champs, dans les vignes ou les vergers pour abriter le matériel, prendre les repas lors des jours de travail, loin du village » (Fénelon 1970). Le terme «cabane » a un sens légèrement différent; il désigne une construction de petite dimension, édifiée avec des matériaux médiocres, et isolée dans un verger ou un vignoble. Dans ce contexte, la cabane sert à abriter les bergers, les chasseurs, les vignerons, les animaux domestiques et le matériel agricole (ibid). On retient de ces deux définitions l'idée d'un bâtiment à vocation agricole, installé à distance de l'espace villageois, de qualité et de facture assez modestes sinon médiocres alors que dans d'autres mondes, urbains notamment, le cabanon est un habitat de loisir ou de repos (Raveneau $\&$ Sirost 2011). Durant la période de l'agnelage d'automne, en plaine, le berger loge à proximité de la bergerie afin de faciliter, la nuit, la surveillance des mises-bas (Figure 5). Plus tard, dans le coussoul, il s'installe dans un autre cabanon lui aussi généralement attenant à la bergerie. Malgré les efforts récents des propriétaires privés (éleveurs, propriétaires fonciers) ou publics (municipalités) et d'une politique d'amélioration de l'habitat pastoral ${ }^{5}$, les conditions de logement sont parfois telles que le berger préfère s'installer dans une caravane. 

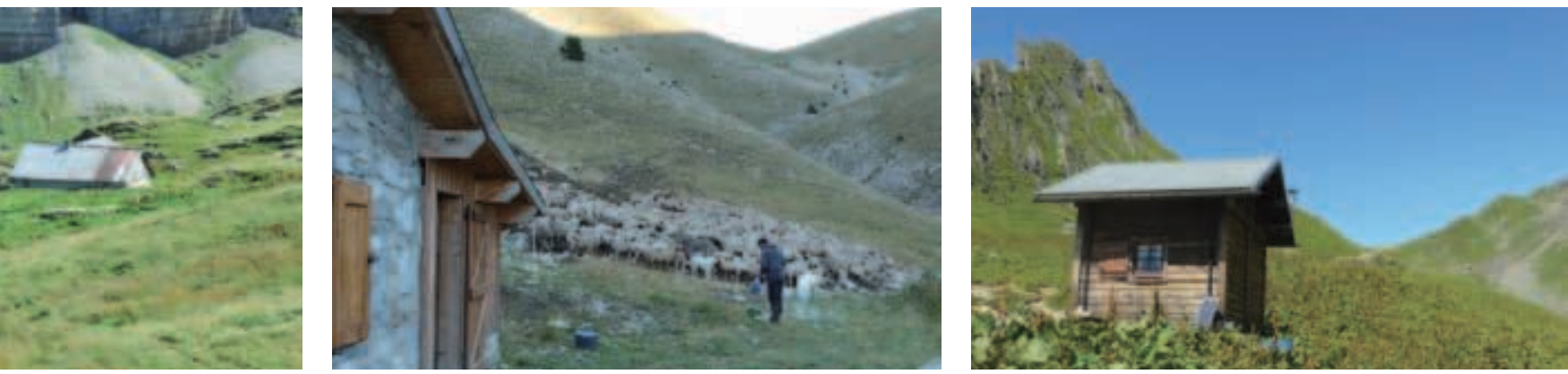

${ }^{\circ}$ M. Meuret - Fig. 8

๑ M. Vincent - Fig. 9

Cette dernière est, du reste, le seul recours en colline où l'absence de logement est la règle. De telles situations ne font pas mentir l'ancienne mauvaise réputation de l'habitat pastoral en plaine par comparaison avec la cabane d'alpage qui était, au début du xx ${ }^{e}$ siècle, décrite comme bien plus agréable (Van Gennep 1999 [1949]).

\section{Chalets (d'altitude) et cabanes (d'alpage)}

Les termes « chalet » et « cabane d'alpage » renvoient à des standards, notamment d'usage et de confort, bien contrastés. Le premier désigne un habitat agricole saisonnier lié à la fabrication fromagère en montagne en système laitier bovin ${ }^{6}$. C'est aussi l'habitat saisonnier des agriculteurs des vallées alpines qui s'y installaient pour exploiter les « petites montagnes » durant quelques mois. Alors que la cabane est synonyme de solitude et d'isolement, renvoyant à un univers masculin et une activité spécialisée (la garde), les chalets formaient souvent des hameaux d'altitude retrouvant chaque année vie pendant plusieurs mois. Ils constituaient le cour de l'exploitation agricole et pastorale (Mallet 1999), comme par exemple les chalets de Furfande et de Clapeyto dans le Queyras ou encore ceux de Vormy dans les Aravis (photo d'ouverture). S'y développait une vie familiale et sociale, avec une main-d'œuvre nombreuse accomplissant une diversité de tâches: foins, bois, pêche et chasse, jardins, garde du troupeau de moutons (Brun 2000). Les cabanes d'alpage, situées plus haut, étaient occupées par le berger du village et/ou par les bergers transhumants qui exploitaient les « grandes montagnes ».

Aujourd'hui, les cabanes se répartissent selon leur taille et leur situation dans l'alpage, entre cabanes principales et cabanes secondaires. La cabane principale, aux alentours de 1500 mètres, est habitée en début d'estive (Figures 6, 7 et 8) et à la redescente du troupeau, en septembre. Il peut s'agir d'un ancien chalet d'altitude réinvesti, et éventuellement rénové. Relativement grande et confortable, c'est là que s'effectuent les rassemblements et les comptages du troupeau ou les soins aux animaux. Elle permet d'utiliser une grande partie de l'alpage, des anciennes prairies de fauche situées tout en bas aux quartiers de juillet dont la repousse est pâturée en septembre. Les quartiers éloignés ou excentrés sont, quant à eux, exploités à partir d'une ou plusieurs cabanes secondaires. Ces bâtiments plus petits et plus haut situés ne sont occupés que le temps nécessaire à «faire manger l'herbe » proche (Figure 9).

\section{Cabane principale d'alpage dans les Alpes-de-Haute-Provence}

Le regroupement nocturne du troupeau dans un parc électrifié proche de la cabane, avec présence de chiens de protection ou patous, permet au berger d'intervenir rapidement en cas d'attaque de loup.

(Fig. 8)

\section{Cabane secondaire en Savoie}

De taille moyenne, située à 1800 mètres d'altitude, elle permet d'exploiter un quartier isolé de l'alpage. Elle est pourvue d'un étage limitant la promiscuité du berger et de son aide. On remarque la présence d'une cheminée et d'un panneau solaire. Les nombreuses plantes nitrophiles (rumex au premier plan) témoignent du parcage fréquent et ancien des troupeaux sur ce site.

(Fig. 9) 


\section{La cabane: lieu de vie et outil de gestion de l'estive}

La vocation de la cabane d'alpage est double. Lieu de vie et de repos pour le berger (et éventuellement ses hôtes ou sa famille), elle est également un véritable outil de gestion de l'estive. Son emplacement et son agencement doivent tenir compte de ces deux dimensions. Sa localisation renvoie à l'organisation spatiale et temporelle des pâturages qui varie selon la structuration de l'estive, l'altitude des secteurs de pâturage, les ressources fourragères, la disponibilité en eau, le relief ou encore de la problématique environnementale. L'ensemble de ces éléments permet d'établir un « diagnostic pastoral » spécifique de chaque estive. Ce document aide à définir l'emplacement pertinent pour la cabane. La gestion du troupeau et des différents quartiers d'alpage influe fortement ce choix qui peut soit privilégier une position centrale par rapport aux différents quartiers d'alpage soit, une situation excentrée permettant l'accès à un quartier éloigné dont l'exploitation à date précise est stratégique dans le cycle d'utilisation de l'ensemble de l'unité pastorale. Ensuite, si le troupeau passe la nuit en « couchade naturelle», c'està-dire dans un lieu qu'il choisit lui-même et où les bêtes ne sont pas parquées, il faut tenir compte du temps nécessaire au berger pour regagner la cabane le soir et retrouver ses brebis le matin. Si le trajet excède une demi-heure, le travail d'alpage peut devenir compliqué, et le troupeau en pâtir (Centre d'Études et de Réalisations Pastorales AlpesMéditerranée \& Institut de l'Élevage 2002). Par ailleurs, dans ces grands espaces au relief tourmenté que sont les estives alpines, les contrats agri-environnementaux imposent aux bergers une gestion fine de la montagne avec des circuits de pâturage contraignants et des déplacements importants. Une bonne répartition des cabanes et de leurs parcs de nuit à travers l'unité pastorale permet au troupeau et au berger de regagner chaque soir leur point de départ - ce qui est imposé par le Plan loup (cf. infra).

Pour des raisons de sécurité et de confort, le site retenu doit être protégé des risques naturels majeurs (avalanche, éboulement), abrité du vent dominant, proche d'un point d'eau potable. L'accès à une voie de communication facilite grandement l'approvisionnement régulier et l'acheminement du matériel nécessaire: bois de chauffage, sel pour les bêtes, aliments pour les chiens, parcs, filets, etc., qui sinon, s'effectuent par héliportage. Il est également préférable que le site soit relativement plat, avec une bonne perspective sur les pâturages alentours et sur le parc nocturne. La cabane n'est pas seulement le point névralgique depuis lequel s'organise le travail et se structurent les déplacements quotidiens, c'est aussi un espace privé à usage domestique, offrant abri et repos au berger. C'est bien toute sa spécificité et sa complexité: combiner les dimensions professionnelles et privées, qui chacune renvoie à des répertoires normatifs, réglementaires ou simplement d'usage propres.

\section{La cabane, un objet sous tension normative}

De la minuscule cabane au toit de tôle, à peine hors d'eau au chalet mezzanine avec panneau solaire, en passant par la simple « cave au sol en terre battue » que certains bergers ont encore très récemment connue (Delahaye \& Picart 1995), l'état des cabanes est très variable. Les conditions de vie s'améliorent sans conteste, les bergers n'acceptant

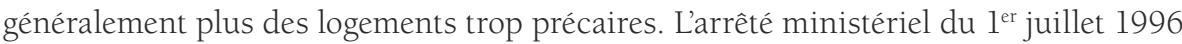
précise les caractéristiques des logements des bergers-vachers d'estive sur la base d'une 
dérogation du décret du 24 août 1995 relatif à l'hébergement des travailleurs agricoles. Les conventions collectives, encore indigentes sur cette question - que le contrat de travail ne prévoit pratiquement jamais - s'en tiennent souvent à quatre des points précisés dans le décret: 100 litres d'eau potable par jour et par personne, $11 \mathrm{~m}^{3}$ minimum par personne pour le sommeil, moyen de cuisson obligatoire et salubrité du bâtiment. Ces critères questionnent la construction de la norme générale (clairement définie pour les travailleurs des filières des fruits et légumes) et pointent les limites de son transfert dans un contexte aussi singulier que celui du logement pastoral en estive. Ils apparaissent en effet à la fois extrêmement indigents, parfois difficilement applicables, voire inadaptés, comme on le verra d'ici peu avec les cabanes d'appoint. Ils révèlent à quel point s'impose un « modèle politique [...] de confort » calqué sur la norme « d'en bas » (Delavigne \& Roy 2002 : 102).

La complexité juridique et le contexte socio-économique de certains alpages peuvent rendre les dossiers délicats et longs à instruire, les délais entre le dépôt d'un dossier de subvention et la réalisation des travaux variant entre trois et douze mois. Lamélioration de ces cabanes a un coût : 2000 à 2500 euros TTC/m² (cf. Direction Régionale de l'Alimentation, de l'Agriculture et de la Forêt et Conseil régional), hors transport, celui-ci s'effectuant souvent par hélicoptère. Des fonds d'État, destinés à financer l'amélioration en matière d'équipements pastoraux (clôtures, cabanes, parcs de contention et de tri, point d'eau), en prévoient le subventionnement. Un dossier éligible à certaines aides peut ainsi être pris en charge à $80 \%$ seuls $20 \%$ incombant alors au maître d'ouvrage. La rénovation, l'agrandissement et la construction de cabanes pastorales sont soutenus d'un point de vue logistique et financier par les services de l'État (DDT) ou d'alpage (chambres d'agriculture) et les équipes techniques des parcs naturels nationaux et régionaux, des réserves nationales et de l'Office National des Forêts (ONF). L'amélioration des cabanes se heurte à une situation paradoxale et problématique : les éleveurs sont responsables du logement de leurs bergers dans un territoire dont ils n'ont généralement ni la maîtrise ni la propriété, les pâturages étant loués (Charmetant 2003). La volonté ou même la seule possibilité d'intervenir est de ce fait parfois restreinte. Enfin, les contraintes juridiques sont complexes étant donné le statut de ces espaces car les projets doivent aussi tenir compte des codes de l'urbanisme et de l'environnement - les zones d'altitude étant souvent des espaces naturels protégés.

Le bon équipement d'une montagne en cabanes pastorales permet de gérer le plus finement possible ces territoires fragiles et riches, et d'y fidéliser un berger ou une bergère. À l'inverse, faute de cabanes décentes, un alpage est voué à l'abandon lorsque le berger attitré qui se satisfaisait encore d'un abri sommaire partira en retraite et sera remplacé par un jeune qui, lui, probablement, n'en voudra pas. Dans d'autres cas, l'alpage est confié chaque été à un nouveau berger - aucun n'étant prêt à y revenir. De tels renouvellements se font au détriment des brebis, du territoire et du salarié puisqu'ils empêchent la capitalisation des savoirs propres à une montagne et compliquent le travail des bergers successifs. Or, l'alpage est une période difficile pendant laquelle les bergers sont seuls face à de très gros troupeaux et des responsabilités accrues liées notamment à l'agri-environnement et à la protection des troupeaux contre le loup dont le retour a souligné l'importance de la cabane et son rôle dans l'organisation du travail pastoral. Un nouvel habitat a dû être inventé: la cabane d'appoint ou cabane prédation. 


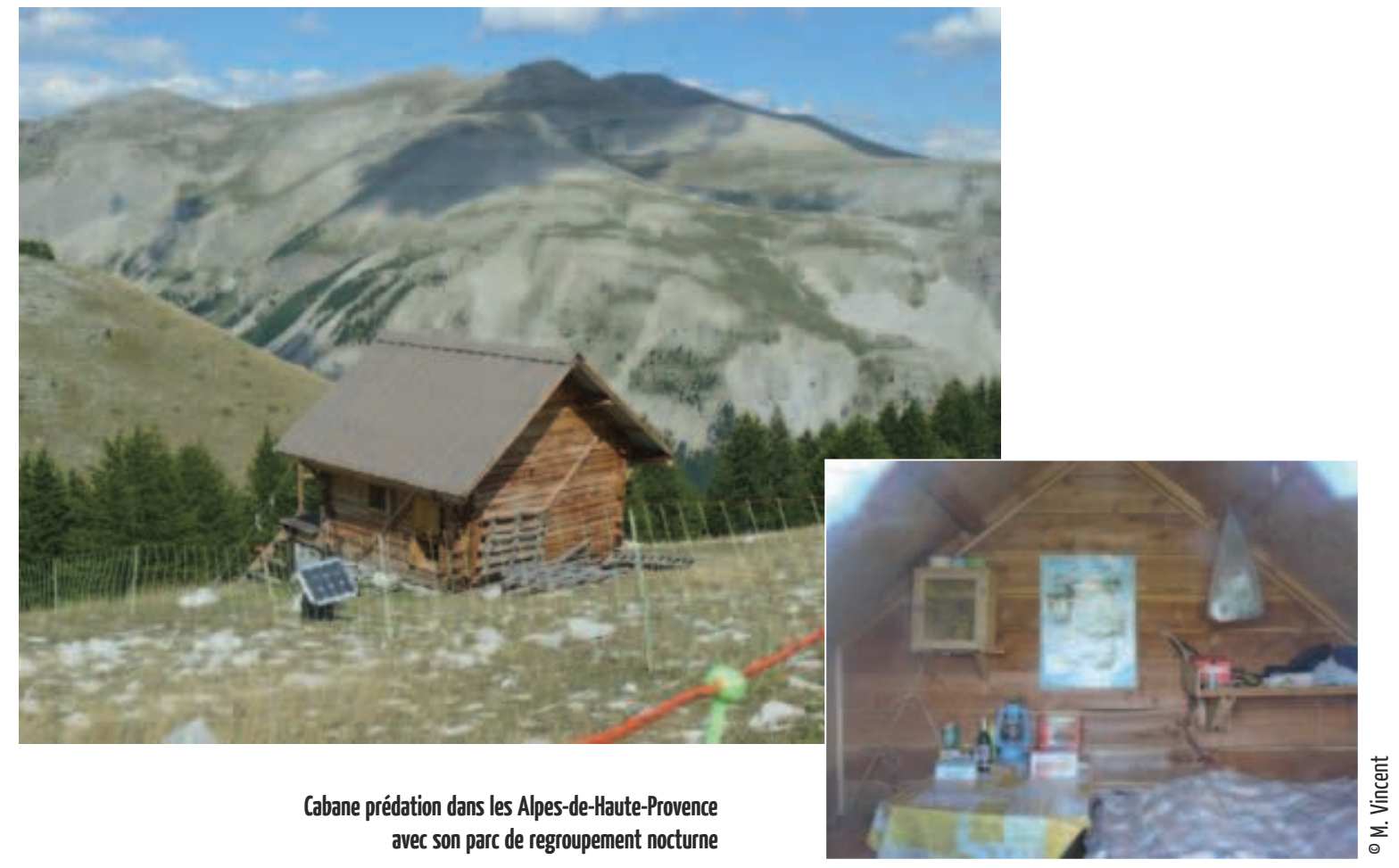

Ce petit bâtiment de $9 \mathrm{~m} 2$ est posé sans fondation ni plateforme à plus de 2000 mètres d'altitude dans une forte pente. Construit en plein courant d'air sur un site qui n'a jamais connu de construction, il s'est avéré indispensable de l'arrimer au sol par de forts câbles. L'appareil d'électrification de la clôture alimente aussi l'éclairage du bâtiment qui n’a toutefois pas de chauffage.

L'unique pièce abrite un lit, une table, un meuble de rangement et un réchaud à gaz (non visible). Noter la lampe tempête nécessaire à la surveillance nocturne du troupeau. Ce logement est peu adapté, de par ses dimensions réduites, à l'accueil d'un berger et d'un aide-berger.

(Fig. 11 et 12)

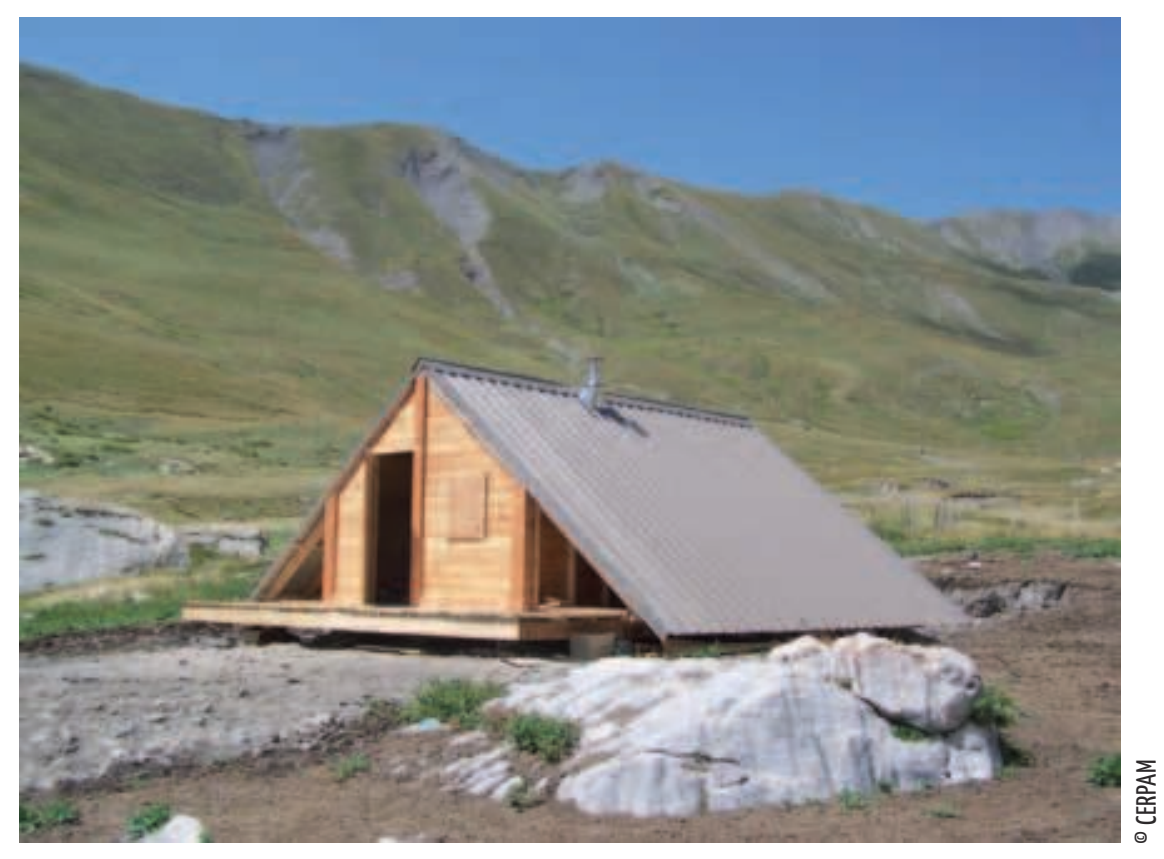

Cabane prédation de conception récente et bien adaptée aux conditions de vie et de travail en alpage

La surface importante de toiture permet de récupérer l'eau de pluie, l'auvent est destiné à stocker du matériel et à abriter le chien de travail, le conduit de fumée témoigne de l'existence d'un système de chauffage. Deux pièces permettent d'y loger convenablement deux personnes. (Fig. 13) 


\section{Un nouveau front de mobilité: les cabanes d'appoint}

En août, le troupeau gagne les secteurs les plus élevés, qui culminent à 2500 mètres environ. Sur ces quartiers d'août, les conditions de vie et de travail sont éprouvantes: la dégradation météorologique et les brouillards sont propices aux attaques. Ordinairement à cette époque, le berger regagne tous les soirs sa cabane située plus bas et laisse ses bêtes passer la nuit sur les crêtes en couchades naturelles (Figure 10-A). Les mesures de protection du troupeau prévues par le Plan loup ${ }^{7}$ imposent le parcage nocturne à proximité de la cabane. De fait, le troupeau doit redescendre chaque soir avec son berger à la cabane et regagner chaque matin ses pâturages d'altitude (Figure 10-B). Non seulement ces nouveaux circuits imposés fatiguent les bêtes, mais ils empiètent également sur leur temps de repas et de repos, tout en désorganisant le plan de pâturage. Par ailleurs, les trajets quotidiens de si grands troupeaux accentuent l'érosion des drailles, tandis que l'accumulation des déjections animales dans les parcs favorise les pathologies des pieds (piétin) et constitue une source de pollution des eaux: autant de dégradations incompatibles avec la vocation agri-environnementale du pastoralisme qui mettent en évidence les contradictions des politiques publiques (Vincent 2009). L'installation sur les quartiers d'août de cabanes d'appoint - dites aussi « cabanes prédation » ou « cabanes d'altitude »-permet de remédier à la situation (Figures 11, 12 et 13). Le berger loge à côté de son troupeau, lequel peut alors, soit garder ses habitudes de couchade libre si la sécurité des bêtes est assurée, soit dormir dans un parc installé à proximité de la cabane d'appoint (Figure 10-C).

\section{Construire en crête}

Désemparés, pris au dépourvu et très affectés par les attaques, certains - rares - bergers ont choisi de dormir sur place dehors auprès de leur troupeau (Lebaudy \& Pourcel 2003-2004; Vincent 2011). Dans l'urgence, des infrastructures, très provisoires et pour le moins inadaptées (caravane, abri de chantier), ont été héliportées et installées à la hâte. L'altitude rend particulières la conception et la construction de cabanes, en dehors même de l'acheminement des matériaux qui nécessite l'héliportage. Le logement n'est occupé que deux ou trois semaines mais doit résister à l'enneigement et offrir l'été les garanties de confort (isolation et chauffage) et de sécurité (risque de foudre) qui s'imposent à ces altitudes. De telles cabanes sont depuis peu disponibles sur le marché. Certaines sont composées de deux pièces (Figure 13). Un tel agencement, encore rare, reflète l'évolution du travail en estive sous l'effet très direct du retour du loup.
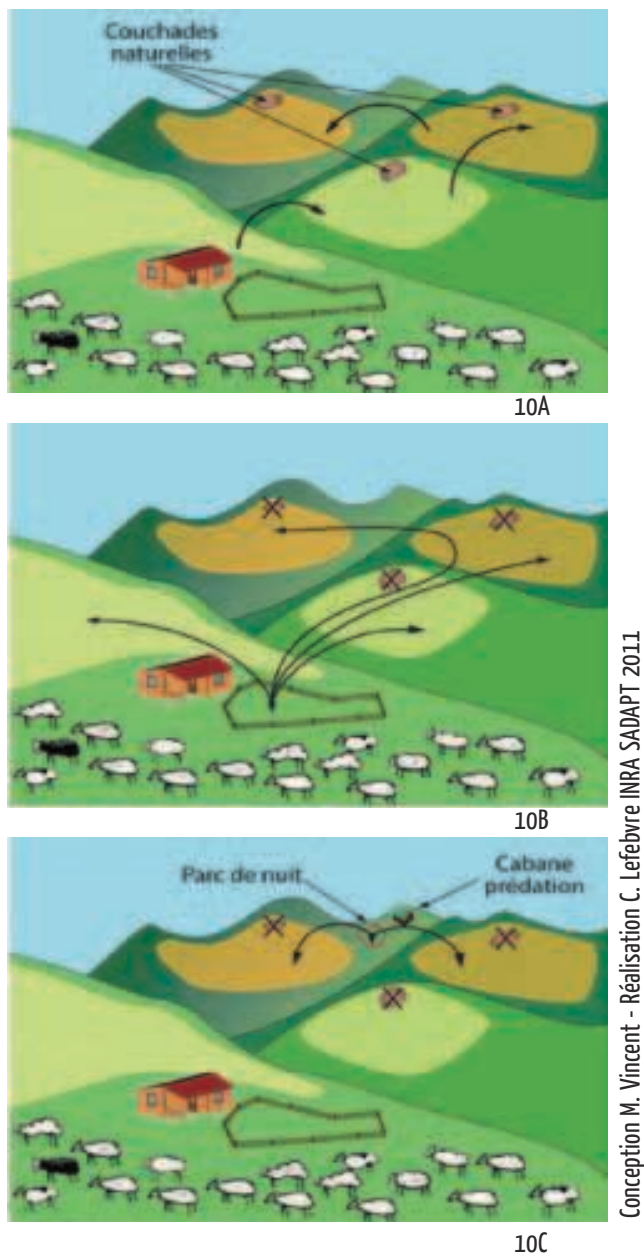

Conséquences du retour du loup sur l'exploitation de l'alpage

A- Avant le retour du loup, les anciennes couchades libres permettaient d'exploiter le plus finement possible les différents quartiers de l'alpage.

B - L'abandon des couchades libres sur les quartiers d'août contraint les brebis à redescendre à la cabane principale ou secondaire tous les soirs. Le cheminement répété des brebis dégrade les ressources fourragères et provoque de l'érosion.

C - Les cabanes d'appoint, ou cabanes « prédation », installées sur les quartiers d'août permettent aux bêtes de rester la nuit sur les crêtes.

(Fig. 10A-10B-10C) 
En effet, aux côtés du berger un autre personnage est apparu. Il s'agit de l'aide-berger dont le salaire est subventionné par le Plan loup, qui prend en charge le surcroît de travail lié à la protection du troupeau: surveillance, pose des clôtures, entretien du chien de protection (le patou), remplacement occasionnel du berger, soit entre six à huit heures de travail quotidien en cas de prédation (Jallet \& Fabre 2007). L'aide-berger est recruté par l'éleveur qui impose donc à son berger un compagnon dans un espace réduit et pour une durée de temps relativement longue. Une telle promiscuité est difficile à imposer et à vivre, surtout si le berger est une bergère - ce qui est de plus en plus souvent le cas. La cohabitation n'est possible que si berger et aide-berger sont apparentés: mari et femme, père et fils... Le Centre d'études et de réalisations pastorales Alpes Méditerranée (Cerpam) propose des bâtiments de surface plus importante et comportant deux pièces $\left(20 \mathrm{~m}^{2}\right)$ afin de mieux cadrer avec la législation sur le logement des ouvriers agricoles. Leur coût au $\mathrm{m}^{2}$ est inférieur à celui des cabanes à une pièce: 1300 euros TTC/m² soit 26000 euros, auxquels il convient d'ajouter $1 / 5^{\mathrm{e}}$ de cette somme pour une demidouzaine d'héliportages et le terrassement.

L'équipement des quartiers d'août en cabanes d'appoint se traduit par l'ouverture d'un nouveau front de mobilité dans des secteurs situés aux confins du territoire jusqu'ici anthropisé. Si les brebis y prennent leurs (hauts) quartiers (de fin) d'été, les bergers ne s'y sont jusqu'ici jamais véritablement établis. La construction de cabanes, occupées très peu de temps, y est d'autant plus nécessaire que le risque de prédation décuple la pénibilité des conditions de travail (froid, brouillards, dénivelé) et de vie (isolement, approvisionnement, hygiène, confort élémentaire). Les contraintes techniques augmentent, qu'il s'agisse de la conception des cabanes (assurant sécurité et durabilité), du choix des matériaux (isolation thermique, panneaux solaires), de leur acheminement sur place ou encore de la procédure à mettre en œuvre pour mener à bien un tel projet. La normalisation de ce type d'habitat d'appoint interroge le modèle normatif en vigueur, déjà parfois difficilement applicable pour les cabanes secondaires. Elle pourrait faire encourir le risque de bloquer des projets de cabanes tout en demeurant inadaptée aux réalités du métier de berger. Par exemple, plutôt que d'imposer cent litres d'eau potable par jour et par personne ou une surface minimale pour le sommeil, la législation pourrait porter sur l'agencement des cabanes de façon à garantir à chacun des salariés son espace privé.

Sans résoudre la question du loup, l'amélioration, l'entretien et la construction de logements adaptés aux réalités actuelles du métier de berger et des territoires montagnards contribuent à minimiser le risque de précarisation professionnelle et à assurer la pérennité et la continuité d'une exploitation pastorale des territoires montagnards. Le retour du loup a contraint à reconsidérer le logement pastoral dont l'importance dans l'entreprise de modernisation de l'économie agricole n'avait pas échappé aux municipalités concernées et aux organisations professionnelles au milieu du xx $x^{e}$ siècle. Ainsi, au sortir de la Seconde guerre mondiale - et même avant - certaines communes alpines et syndicats d'élevage se sont distingués pour leurs efforts dans ce domaine, apportant ici « l'appareil butagaz » permettant de « résoudre le problème du chauffage de l'eau, de la cuisson des aliments d'une manière commode et rapide » (Rogié 1951-52 : 191), rénovant, nettoyant, isolant et agrandissant, là, de vieilles cabanes, en construisant ici de nouvelles, ou installant ailleurs des « baraques Adrian » offertes par l'armée. "Les temps sont révolus des esprits purs et simples acceptant de vivre avec leurs bêtes, comme Diogène, non pas dans un tonneau mais sous une pierre ", 
rappelle en 1960 la Société d'économie alpestre (Rogié 1959-1960: 630) qui, cette année-là, a décerné le premier prix du concours d'alpage à la commune d'Orcières pour son programme de « travaux d'améliorations pastorales » exécuté entre 1925 et 1936.

À chaque époque, sa «modernisation ». Le $\mathrm{xxI}^{\mathrm{e}}$ siècle de la montagne s'ouvre sur une gageure de taille, la nécessité de faire cohabiter pastoralisme et loups. Mais un biais apparaît: en repoussant les limites de l'habitat vers les cimes, la sécurité des brebis se paye par l'assujettissement du berger pendant que le loup court toujours. Et ce faisant, l'obligation d'installer un logement dans les secteurs d'alpage les plus isolés ne peut que laisser craindre qu'au caractère très temporaire de ce type d'habitat ne vienne s'ajouter une forte précarité dans des territoires où, précisément, l'exercice du métier de berger exige non pas uniquement la sécurisation du seul troupeau mais aussi celle des hommes au travail. Pointer un tel risque, tel est l'éclairage que nous pouvons verser à la réflexion générale développée dans ce volume.

\section{NOTES}

Photo d'ouverture: Chalets d'alpage de Vormy regroupés en hameau pour la traite des vaches. Massif des Aravis, Haute-Savoie.

1. Soit en moyenne 750 bêtes, contre 250 en France.

2. Naissance des agneaux

3. Il s'agit de brebis qui ne sont ni gestantes, ni allaitantes.

4. Le berger de métier dispose d'un logement permanent en plaine à proximité du siège de l'exploitation qui l'emploie. En revanche, les plus jeunes, qui ne sont pas encore stabilisés professionnellement, sont parfois logés de façon temporaire à la morte saison.

5. Voir la campagne d'électrification de vingt-cinq bergeries portée par Espaces naturels de Provence, avec le soutien des conseils régionaux et généraux, de l'ADEM, d'EDF et de la CEE, en 1995 (Delahaye $\&$ Picart 1995)

6. Une autre forme de mobilité est attestée en Vanoise où, au début du xx ${ }^{\mathrm{e}}$ siècle, se pratiquait le système de l'arbé, lieu de fabrication itinérante du Beaufort. Il s'agissait d'un bâtiment sans toit permanent; celui-ci était de bâches et de planches afin de pouvoir être déplacé régulièrement de site en site de façon à coller au plus près de la pousse de l'herbe. Personnel, troupeau, cochon et chaudron faisaient alors partie de la caravane (Brugière 2000).

7. L'État a mis en place dès 1993 un dispositif qui vise à « garantir un état de conservation favorable du loup » et « réduire les dommages aux troupeaux ». Ce dispositif souvent appelé «Plan loup » est devenu le « Plan d'action sur le loup » à partir de 2004. Relayé en 2008 par le « Plan d'action national sur le loup », il prévoit trois mesures de protection des troupeaux et les subventionne: l'aide-berger, le chien de protection (le patou) et le regroupement nocturne des moutons dans un parc électrifié à côté d'une cabane (MEEDDAT \& MAP 2008). 


\section{RÉFÉRENCES}

Badan, O. Brun, J.-P. \& Congès, G. 1996 Les Bergeries romaines de la Crau d'Arles. Les origines de la transhumance en Provence, Gallia 52: 263-310.

Brisebarre, A.-M. 2007 Bergers et transhumance. Paris: Édition De Borée.

Brugière, Y. 2000 Le Système de l'arbé en Vanoise. In B. Brun, A.H. Dufour \& al. (dir.) Cabanes, cabanons et campements. Formes sociales et rapports à la nature en habitat temporaire. Bordeaux: Société d'écologie humaine, 123-131.

Brun, B. 2000 La Cabane et l'écologie humaine. In B. Brun, A.H. Dufour \& al. (dir.) Cabanes, cabanons et campements. Formes sociales et rapports à la nature en habitat temporaire. Bordeaux: Société d'écologie humaine, 9-18.

Centre d'Études et de Réalisations Pastorales Alpes Méditerranée \& Institut de l'Élevage 2002 Cabanes pastorales d'alpages dans les Alpes du Sud. Manosque: CERPAM (Techniques pastorales).

Charmetant, R. 2003 Mise en place d’un référentiel méthodologique "de projet " sur les cabanes pastorales en Languedoc-Roussillon. DAA d'agro-environnement: Agro Montpellier.

Claudot-Hawad, H. 2002 Touaregs. Apprivoiser le désert. Paris: Gallimard (Découvertes), nº 418.

Conservatoire Études et Écosystèmes de Provence \& Chambre d'Agriculture des Bouches-du-Rhône 1997 Patrimoine naturel et pratiques pastorales en Crau. Pour une gestion globale de la plaine. CEEP, CA 13, CERPAM, AOM, INRA (Dir.).

Delahaye, A. \& Picart, E. 1995 L'Équipement des cabanes de berger dans les Alpes du Sud. Projet d'ingénieur: Agro Montpellier (2 vol.).

Delavigne, A.-E. \& Roy, F. 2004 La Vie privée du berger. Incursions cinématographiques dans l'habitat de bergers transhumants en estive, Revue de géographie alpine 92 (3): 95-102.

Fabre, P. \& Lebaudy, G. 2010 Le Mérinos d'Arles. Passion de bergers. Marseille: Images en Manœuvres \& Maison de la Transhumance.

Faye, B. 2008 Bergers du Monde. Versailles: QUÆ.

Fénelon, P. 1970 Vocabulaire de géographie agraire. Paris: Armand Colin.

Jallet, M. \& Fabre, P. 2007 Organisation du travail face à la prédation: redéfinition des métiers d'alpage. In Cerpam, Institut de l'Élevage \& Sime (Dir.), Actes du séminaire technique Loup/Élevage. S’ouvrir à la complexité... Aix-en-Provence, 15 et 16 juin 2006: 108-116.

Lebaudy, G. \& Pourcel, F. 2003-2004 Cabanéjer, aménager, décorer... : Cabanes, cabanons, maisons des bergers transhumants de la Crau (Bouches-du-Rhône). Rapport d'enquête pour l'exposition «Bergers de Crau: au-delà de l'image ». Arles: Muséon Arlaten.

Legeard, J.-P. 1996 Bergers d'alpages. Résultats d'enquête auprès des employeurs et salariés en fin d'estive. Manosque : CERPAM.

Legeard, J.-P. \& al. 2010 Où en sont les bergers aujourd'hui? In M. Meuret (Dir.), Un savoir-faire de bergers. Dijon et Versailles: Educagri \& QUÆ.

Mallet, M.-P. 1999 Habitat permanent, habitat saisonnier. In Association pour le patrimoine de Provence \& Société d'études des Hautes-Alpes (Dir.) L'Habitat du nord des Hautes-Alpes: patrimoine architectural et mobilier. Aix-en-Provence: Cahiers du Patrimoine, 83-103.

Ministère de l'Écologie, de l'Énergie, du Développement Durable et de l'Aménagement du Territoire et Ministère de l'Agriculture et de la Pêche (MEEDDAT \& MAP) 2008 Plan d'action national sur le loup 2008-2012, dans le contexte français d'une activité importante et traditionnelle d'élevage, 3 juin 2008.

Raveneau, G. \& Sirost, O. 2011 Anthropologie des abris de loisirs. Paris: Presses Universitaires de Paris X. 
Rogié, J.-P. 1951-1952 Compte rendu du concours d'alpage de 1951, Bulletin de la fédération française d'économie alpestre 2: 191.

- 1959-1960 Compte rendu du concours d'alpage de 1959, Bulletin de la fédération française d'économie alpestre 10: 630 .

Van Gennep, A. 1999 [1949] Cérémonies périodiques, cycliques et saisonnières, IV. Paris: Robert Laffont.

Vincent, M. 2009 Le pastoralisme méditerranéen sous l'effet des politiques contradictoires de l'agrienvironnement et du loup. Ethnozootechnie 87 - Varia 9: 167-168.

— 2011 Les Alpages à l'épreuve des loups. Versailles: QUÆ. 


\section{RÉSUMÉ}

De Cabanons en cabanes: habitat pastoral et retour du loup. L'élevage de Crau pour exemple. Dans le Sud-Est de la France, le pastoralisme repose sur l'exploitation successive de différents pâturages qui permettent de disposer tout au long de l'année de ressources fourragères mangées sur pied par les troupeaux. Un tel système est fondé sur l'itinérance et le gardiennage quotidien des bêtes par un berger qui suit le troupeau dans ses déplacements annuels, dont l'ampleur est variable. La transhumance, par laquelle hommes et bêtes quittent, à la fin du printemps, la plaine pour les pâturages alpins, s'inscrit dans ce système d'économie fourragère qui caractérise de nombreuses cultures pastorales de Méditerranée et d'ailleurs. Cette organisation suppose une mobilité résidentielle que peu d'études questionnent et dont nous proposons une première approche à partir de l'élevage ovin transhumant de la Crau. On s'attache à décrire et analyser les différents habitats des bergers transhumants, du cabanon de Crau à la cabane d'alpage, dans un contexte de transformation forte du métier de berger. Celle-ci tient, d'une part, à l'évolution des caractéristiques sociologiques (féminisation, rajeunissement) d'une activité en voie de professionnalisation (formation et qualification accrue, légitimité environnementale). Elle tient d'autre part à l'installation du loup, depuis 1992, dans les territoires pastoraux alpins. Cet événement a considérablement bouleversé les fondements du pastoralisme qui s'était développé en l'absence de ce prédateur, dont le retour a très brutalement imposé une réorganisation des pratiques d'élevage en montagne. C'est dans ce contexte très particulier que nous examinons les formes et les modalités de la mobilité résidentielles des bergers tout au long de l'année, en nous focalisant particulièrement sur l'habitat en montagne. En effet, nous montrons en quoi la présence du loup rend encore plus décisive et stratégique la double fonction, résidentielle et pastorale, des cabanes d'alpage dont une version directement liée à la situation - les cabanes d'appoint ou cabanes " prédation »-commence à s'installer dans le paysage à des altitudes qui n'ont jamais connu la moindre construction.

\section{ABSTRACT}

From Hut to cabin: pastoral housing and the return of the wolf. The example of sheep breeding in the Crau. In the south eastern regions of France, pastoralism is based on the successive exploitation of different pastures allowing for all year round use of fodder resources through grazing. Such a system is founded on itinerancy by a shepherd who looks after the animals on a daily basis and follows them in their yearly changes of location, the scale of which is variable. The transhumance, during which men and animals leave the plains for alpine pastures at the end of spring, forms part of the fodder economy system, which characterises many pastoral cultures around the Mediterranean and elsewhere. Such an organisation implies the residential mobility of shepherds which few studies have so far questioned and which we propose to consider for the first time using the example of ovine transhumant breeding in the Crau. We focus on describing and analysing the various houses of transhumant shepherds, from the Crau hut [cabanon] to the cabin [cabane] in alpine pastures, set in the context of great changes to the shepherds' occupation. These changes result on the one hand from the evolution of the sociological features (increase in women and in younger people) of an activity which is becoming a full professional occupation (training and increasing qualification, environmental legitimacy). On the other hand, they result from the return of wolves to the alpine pastures since 1992. This has considerably altered the foundations of pastoralism, which had developed in the absence of the predator, the return of which has very brutally imposed a reorganisation of breeding practices in the mountains. Our examination of the forms and conditions of shepherds' yearly residential mobility is set against this particular context and focusses on mountain habitats. As we demonstrate, the wolf's presence makes the double function, i.e. residential and pastoral, of alpine cabin even more crucial and strategic, in that a specific type of cabin especially adapted to these new circumstances - namely 'auxiliary' or 'predation' cabin - is starting to appear at altitudes where man-made structures had never before been seen. 


\section{MOTS CLÉS}

Pastoralisme ovin, transhumance, Crau, Alpes, cabane pastorale, loup

\section{KEYWORDS}

Ovine pastoralism, transhumance, Crau, Alpes, pastoral hut, wolf

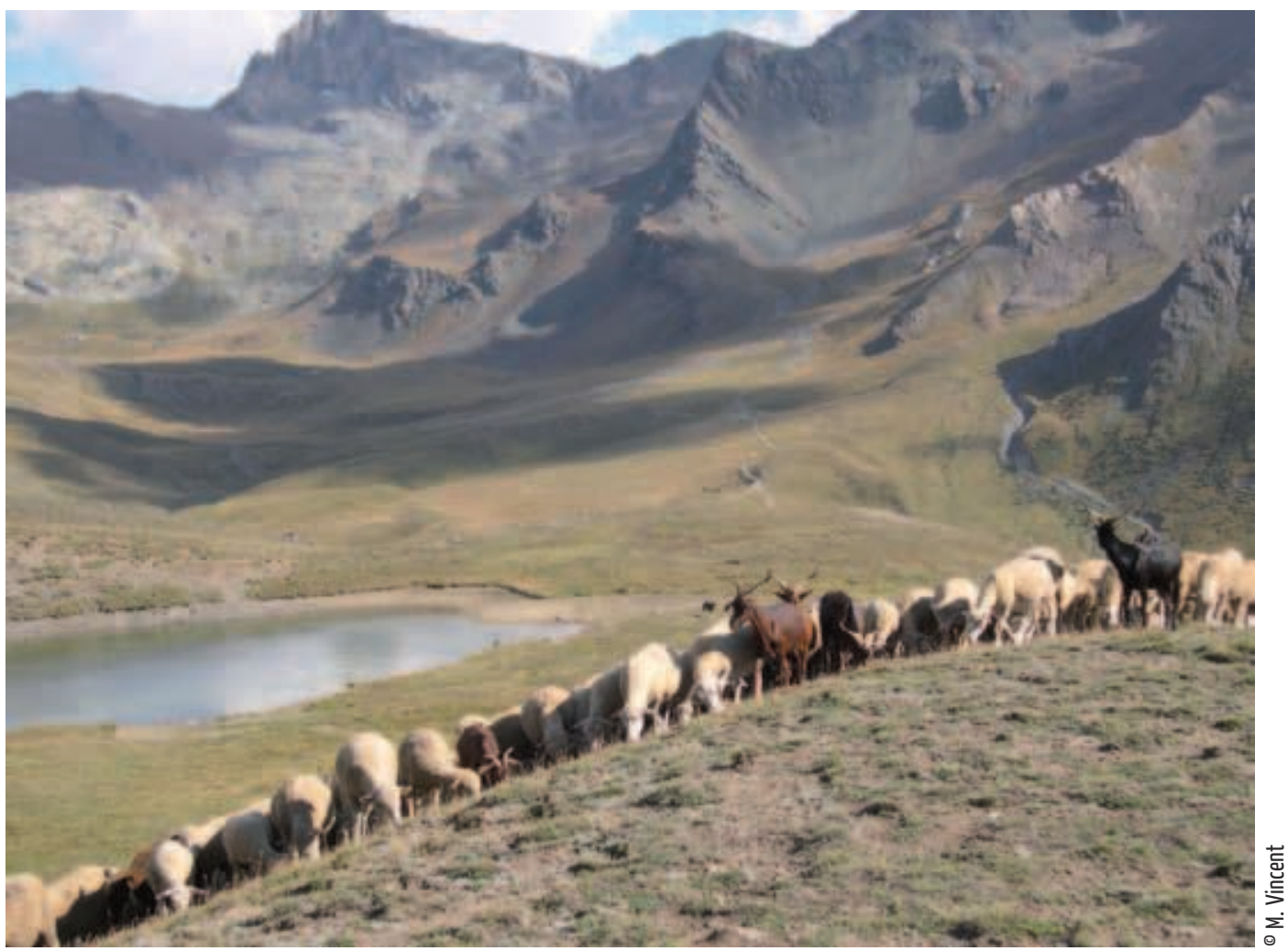

